\title{
Diagnostic Accuracy of High-Resolution Computed Tomography of Chest in Diagnosing Sputum Smear Positive and Sputum Smear Negative Pulmonary Tuberculosis
}

\author{
Waqas Rasheed ${ }^{1}$, Ruby Qureshi ${ }^{2}$, Naila Jabeen ${ }^{3}$, Hyder A. Shah ${ }^{3}$, Rashid Naseem Khan ${ }^{4}$ \\ 1. Pulmonology, Liaquat College of Medicine \& Dentistry, Darul Sehat Hospital, Karachi, PAK 2. Radiology, South City \\ Hospital, Karachi, PAK 3. Radiology, Dow University of Health Sciences, Karachi, PAK 4. Internal Medicine, Liaquat \\ College of Medicine \& Dentistry, Darul Sehat Hospital, Karachi, PAK
}

Corresponding author: Waqas Rasheed,dr.waqasrasheed@gmail.com

\section{Abstract \\ Introduction}

Pulmonary tuberculosis (PTB) is caused by species of organisms in the Mycobacterium tuberculosis complex. It is a major public health problem worldwide and is endemic in Pakistan. Various clinical and biochemical markers exist for its diagnosis. Radiology has an important role in the diagnosis of PTB. Initially, a chest radiograph is warranted for PTB evaluation. High-resolution computed tomography (HRCT) also has high sensitivity and specificity for PTB diagnosis. Features of primary TB include consolidation, lymphadenopathy, pleural effusion and miliary nodules whereas post-primary TB include apical consolidation, nodules and cavitation. The aim of this study was to determine the diagnostic accuracy of HRCT chest in diagnosing sputum smear positive and smear negative PTB.

\section{Methods}

A cross-sectional study was conducted at a large tertiary care teaching hospital. A retrospective review of medical records of patients who underwent HRCT chest and sputum acid-fast bacillus (AFB) direct smear and AFB culture for suspicion of PTB was undertaken. All HRCT chest examinations were performed on multislice computed tomography (CT) scanner. On HRCT, PTB was defined as the presence of consolidation, centrilobular nodules, branching nodules with tree in bud appearance with or without lymphadenopathy and pleural effusion. Diagnostic accuracy of HRCT including sensitivity, specificity, positive and negative predictive values was calculated using $2 \times 2$ table, taking findings of AFB culture as a gold standard.

\section{Results}

A total of 108 patients were included in this study with a mean age of $51.85 \pm 16.86$ years. Diagnostic accuracy of HRCT in diagnosing PTB was found to be $84.26 \%$ with sensitivity, specificity, positive predictive

Received 05/17/2020 Review began 05/23/2020 Review ended 05/26/2020 Published 06/05/2020

() Copyright 2020

Rasheed et al. This is an open access article distributed under the terms of the Creative Commons Attribution License CC-BY 4.0., which permits unrestricted use, distribution, and reproduction in any medium, provided the original author and source are credited. value (PPV) and negative predictive value (NPV) of $89.09 \%, 79.25 \%, 81.67 \%$, and $87.50 \%$, respectively. In sputum smear positive patients, HRCT has diagnostic accuracy, sensitivity, specificity, PPV and NPV of 87.50\%, 88.57\%, 84.62\%, 93.94\%, and 73.33\%, respectively. In sputum smear negative patients, HRCT has diagnostic accuracy, sensitivity, specificity, PPV and NPV of 81.67\%, 90.00\%, 77.50\%, 66.67\%, and 93.94\%, respectively.

\section{Conclusion}

HRCT has high sensitivity in diagnosing sputum smear positive and sputum smear negative PTB. The specificity of HRCT in diagnosing sputum smear positive PTB was high, whereas it was slightly low in diagnosing sputum smear negative PTB. Overall diagnostic accuracy of HRCT was high in diagnosing PTB.

Categories: Radiology, Infectious Disease, Pulmonology

Keywords: sputum smear positive, pulmonary tuberculosis, sputum smear negative, high resolution computed tomography

\section{Introduction}

Tuberculosis (TB) is the infection caused by species of organisms in Mycobacterium tuberculosis complex. Mycobacterium tuberculosis is the major cause followed by other mycobacteria. However, infection is usually not seen in all the exposed persons. Pulmonary tuberculosis (PTB) is a major public health problem as it affects almost one-third of the population of the world [1]. Pakistan is a developing country where tuberculosis is endemic. In developed countries, immigrants from endemic regions are responsible for increasing PTB incidence [2]. Active PTB usually presents with a cough, which is productive with or without hemoptysis, loss of weight, fever with night sweats, and generalized weakness [3]. 
Various tests have been developed for PTB diagnosis. In areas with limited resources, in-house real-time polymerase chain reaction (PCR) could be employed with high specificity and sensitivity [4]. C reactive protein (CRP) is a marker of inflammation and carries high sensitivity for screening active PTB [5]. The Xpert mycobacterium tuberculosis/rifampicin (Xpert MTB/RIF) is a World Health Organization (WHO) approved test for the detection of tuberculosis as well as rifampicin resistance and carries high sensitivity and specificity for diagnosing pulmonary and extra-PTB [6]. It also has a high sensitivity in diagnosing PTB in children [7]. Recently Xpert MTB/RIF Ultra has been introduced and has shown promising sensitivity for diagnosing PTB [8].

An important role of radiological imaging exists in the evaluation of PTB. Initially, a chest radiograph is warranted for the evaluation of PTB. Primary tuberculosis manifests as consolidation, enlarged lymph nodes, pleural effusion and miliary nodules on imaging [9]. Apical consolidation, nodules and cavitation are usually seen in post-primary tuberculosis [3]. It has been suggested that adults in developed countries usually develop primary tuberculosis and in adults of PTB endemic countries, post-primary tuberculosis may occur in response to infection by a second mycobacterium strain [3,10]. According to a study, high-resolution computed tomography (HRCT) chest in smear positive patients has a sensitivity and specificity of $90.9 \%$ and 96.4\%, respectively, in diagnosing active PTB [11]. It is important to utilize accurate means for diagnosing PTB. Therefore, this study was conducted with the aim to determine the diagnostic accuracy of HRCT chest in diagnosing sputum smear positive and smear negative PTB, taking findings of acid-fast bacillus (AFB) culture as the gold standard.

\section{Materials And Methods}

This was a cross-sectional study conducted at a tertiary care teaching hospital. A retrospective review of medical records of patients who underwent HRCT chest and sputum for AFB direct smear and AFB culture for suspicion of PTB was undertaken from January 2018 till December 2019. Both adult male and female patients suspected to have PTB were included. Patients were suspected of having tuberculosis if they presented with productive cough with or without hemoptysis, shortness of breath, fever with night sweats or weight loss. Patients on anti-tuberculous therapy (ATT) were excluded. Patients were also excluded if they were not able to produce sputum. Optimal volume of $2 \mathrm{ml}$ was considered adequate for the sputum smear test.

On HRCT, tuberculosis was defined as the presence of consolidation, centrilobular nodules, branching nodules with tree in bud appearance with or without lymphadenopathy, and pleural effusion. AFB culture was performed on Löwenstein-Jensen (LJ) medium. If there was a growth of mycobacteria then the result was positive and if no growth was seen then the result was negative. Statistical Package for Social Sciences (SPSS) version 20.0 (IBM Corp., Armonk, NY) was used for data entry and analysis. Continuous variables such as age were mentioned as mean and standard deviation. Categorical variables like gender, findings of sputum smear test, HRCT, and AFB culture were mentioned as frequency and percentage. Diagnostic accuracy of HRCT including sensitivity, specificity, positive predictive value (PPV) and negative predictive value (NPV) was calculated using 2 x 2 table taking findings of AFB culture as the gold standard. Stratification was done on the basis of sputum smear result and after stratification sensitivity, specificity, PPV, NPV and diagnostic accuracy were calculated.

\section{Results}

A total of 108 patients were included in this study with a mean age of $51.85 \pm 16.86$ years. Out of 108 patients, a total of 64 (59.3\%) were males and 44 (40.7\%) were females. A total of 60 (55.6\%) patients had sputum smear negative and 48 (44.4\%) had sputum smear positive. These characteristics are summarized in Table 1. 


\section{Cureus}

Baseline characteristics of the patients $(n=108)$

n

Age, years

Gender

Males

Females
64

44

48

60
$\%$

$51.85 \pm 16.86^{\ddagger}$

Sputum smear test

Positive

Negative

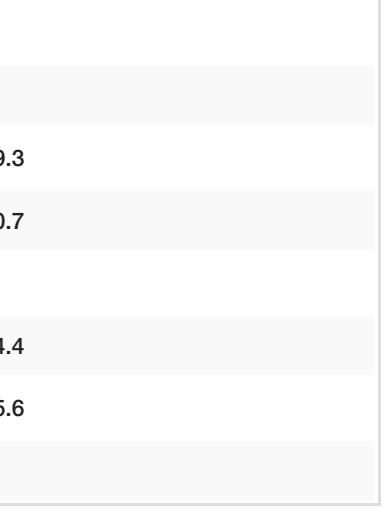

\section{TABLE 1: Baseline characteristics of the patients}

$\ddagger_{\text {Mean }} \pm \mathrm{SD}, \mathrm{n}$ : number

Among 108 patients, positive findings on HRCT were seen in 60 (55.6\%) patients and positive findings on AFB culture were seen in 55 (50.9\%) patients (Table 2).

\begin{tabular}{|c|c|c|c|}
\hline \multicolumn{4}{|c|}{ HRCT chest and AFB culture findings $(n=108)$} \\
\hline \multirow{2}{*}{ HRCT chest findings } & \multicolumn{2}{|c|}{ AFB culture findings } & \multirow{2}{*}{ Total } \\
\hline & Positive & Negative & \\
\hline Positive & 49 & 11 & 60 \\
\hline Negative & 6 & 42 & 48 \\
\hline Total & 55 & 53 & 108 \\
\hline
\end{tabular}

\section{TABLE 2: HRCT chest and AFB culture findings}

HRCT, high-resolution computed tomography; AFB, acid-fast bacillus

Diagnostic accuracy of HRCT in diagnosing PTB was found to be $84.26 \%$ with sensitivity, specificity, PPV and NPV of $89.09 \%, 79.25 \%, 81.67 \%$, and $87.50 \%$, respectively. In sputum smear positive patients, HRCT had diagnostic accuracy, sensitivity, specificity, PPV and NPV of 87.50\%, 88.57\%, 84.62\%, 93.94\% and $73.33 \%$, respectively. In sputum smear negative patients, HRCT had diagnostic accuracy, sensitivity, specificity, PPV and NPV of $81.67 \%, 90.00 \%, 77.50 \%, 66.67 \%$, and $93.94 \%$, respectively.

\section{Discussion}

Any organ system of the body can be affected by tuberculosis and lungs are the most common site usually involved. Sputum smear is often used initially for PTB evaluation. Sputum smear results may take several days to come and the results of AFB culture may take several weeks [12]. Therefore, imaging has an important role in PTB evaluation. In addition to diagnosis, imaging may evaluate treatment response and may also detect any complications associated with this illness [12].

PTB is differentiated into primary and post-primary $\mathrm{TB}$, having different radiological features with slight overlapping. Primary TB may infect lung parenchyma, lymph nodes, pleura or tracheobronchial tree. The focus of primary TB within parenchyma is Ghon focus and together with enlarged lymph node constitutes Ghon/Ranke complex [13]. Post-primary PTB occurs in previously infected/sensitized patients via reinfection/re-activation and this may be due to malnutrition or immunosuppression [12].

PTB is a curable disease, which is endemic in Pakistan. The gold standard for PTB diagnosis is AFB culture. 
One potential diagnostic challenge is also sputum smear negativity with a suspicion of PTB. Various biochemical tests are available that are usually not widely available. Therefore, HRCT can aid in diagnosis and initiating empirical treatment. Moreover, a normal study can help in the exclusion of disease process.

The present study was conducted to evaluate diagnostic accuracy, including sensitivity, specificity, PPV, and NPV of HRCT chest in diagnosing sputum smear positive and smear negative PTB. The study excluded patients undergoing anti-tuberculous treatment. Findings of AFB culture were taken as the gold standard.

Our study has shown that HRCT has a sensitivity of $88.57 \%$ and $90.00 \%$ in diagnosing sputum smear positive and smear negative PTB. Previous studies have shown a high sensitivity of HRCT in diagnosing PTB. A previous study has shown a sensitivity of $96.4 \%$ in diagnosing smear positive active PTB, which was higher as compared to our study [11]. Another study has shown the sensitivity of $82.7 \%$ in diagnosing smear negative PTB, which was slightly lower than the one as compared to our study [14]. Epidemiological differences in PTB prevalence and risk factors could be a possible factor in this difference of sensitivities. Moreover, another potential reason could be a difference in scanning parameters of HRCT.

According to the results of our study, HRCT has a high specificity of $84.62 \%$ for diagnosing sputum smear positive PTB. Whereas, the specificity for detecting sputum smear negative PTB was $77.50 \%$. A previous study has shown a high specificity of 90\% in diagnosing PTB [15]. Another study showed that HRCT has a specificity of $70.5 \%$ for diagnosing smear negative PTB, which was lower than the one reported in our study [16]. For sputum smear positive PTB, a study has shown a higher specificity as compared to our study for diagnosis [11].

HRCT has shown a high NPV for diagnosing sputum smear positive and smear negative PTB. According to our study, PPV of HRCT for diagnosing sputum smear positive PTB was high, whereas it was low for diagnosing sputum smear negative PTB.

An alternative to HRCT is MRI and it is being increasingly studied as a radiation-free tool for diagnosing PTB. A study was conducted on 50 PTB patients that were proven by culture. Both HRCT and MRI were studied. The study results showed that both MRI and HRCT identified pulmonary abnormalities associated with tuberculosis in all the patients [17]. Moreover, MRI had a high resolution that identified caseation, nodal, pleural or parenchymal inhomogeneity better than HRCT [17].

Studies have also shown that radiological manifestation combined with serological testing can help improve diagnosis of PTB. Authors from a previous study [16] evaluated HRCT and interferon-gamma releasing assay (IGRA) for sputum smear negative PTB. The study concluded that combined HRCT and IGRA improve the detection of PTB in cases of sputum smear negativity and help in management by the initiation of treatment early [16].

A study was carried out in the population of Uganda regarding the use of Gene Xpert and chest radiograph in patients with smear negative PTB. The study was carried out on 123 adult patients. The study concluded that a single Xpert test did not yield a positive result in the majority of sputum smear negative PTB. Moreover, chest radiograph over-estimated the PTB in smear negative cases [18].

A potential limitation of this study was its small sample size. Moreover, another limitation of this study was that it was carried out in a population of PTB endemic countries. It is recommended that further studies of multicentric nature should be carried out on a larger sample size to validate the results of this study.

\section{Conclusions}

HRCT has high sensitivity in diagnosing sputum smear positive and sputum smear negative PTB. The specificity of HRCT in diagnosing sputum smear positive PTB was high, whereas it was slightly low in diagnosing sputum smear negative PTB. Overall diagnostic accuracy of HRCT was high in PTB diagnosis. HRCT had low PPV in diagnosing sputum smear negative PTB, whereas high PPV in diagnosing sputum smear positive PTB.

\section{Additional Information}

\section{Disclosures}

Human subjects: Consent was obtained by all participants in this study. Liaquat College of Medicine and Dentistry issued approval IRB-09/LCMD/03/2020. Animal subjects: All authors have confirmed that this study did not involve animal subjects or tissue. Conflicts of interest: In compliance with the ICMJE uniform disclosure form, all authors declare the following: Payment/services info: All authors have declared that no financial support was received from any organization for the submitted work. Financial relationships: All authors have declared that they have no financial relationships at present or within the previous three years with any organizations that might have an interest in the submitted work. Other relationships: All authors have declared that there are no other relationships or activities that could appear to have influenced the submitted work. 


\section{References}

1. Lönnroth K, Raviglione M: Global epidemiology of tuberculosis: prospects for control. Semin Respir Crit Care Med. 2008, 29:481-491. 10.1055/s-0028-1085700

2. Cain KP, Benoit SR, Winston CA, Mac Kenzie WR: Tuberculosis among foreign-born persons in the United States. JAMA. 2008, 300:405-412. 10.1001/jama.300.4.405

3. Nachiappan AC, Rahbar K, Shi X, et al.: Pulmonary tuberculosis: role of radiology in diagnosis and management. RadioGraphics. 2017, 37:52-72. 10.1148/rg.2017160032

4. Wei Z, Zhang X, Wei C, et al.: Diagnostic accuracy of in-house real-time PCR assay for Mycobacterium tuberculosis: a systematic review and meta-analysis. BMC Infect Dis. 2019, 19:701. 10.1186/s12879-0194273-Z

5. Yoon C, Chaisson LH, Patel SM, Allen IE, Drain PK, Wilson D, Cattamanchi A: Diagnostic accuracy of Creactive protein for active pulmonary tuberculosis: a meta-analysis. Int J Tuberc Lung Dis. 2017, 21:10131019. 10.5588/ijtld.17.0078

6. Rakotoarivelo R, Ambrosioni J, Rasolofo V, et al.: Evaluation of the Xpert MTB/RIF assay for the diagnosis of smear-negative pulmonary and extrapulmonary tuberculosis in Madagascar. Int J Infect Dis. 2018, 69:20-25. 10.1016/j.ijid.2018.01.017

7. Detjen AK, DiNardo AR, Leyden J, et al.: Xpert MTB/RIF assay for the diagnosis of pulmonary tuberculosis in children: a systematic review and meta-analysis. Lancet Respir Med. 2015, 3:451-461. 10.1016/S22132600(15)00095-8

8. Dorman SE, Schumacher SG, Alland D, et al.: Xpert MTB/RIF Ultra for detection of Mycobacterium tuberculosis and rifampicin resistance: a prospective multicentre diagnostic accuracy study. Lancet Infect Dis. 2018, 18:76-84. 10.1016/S1473-3099(17)30691-6

9. Burrill J, Williams CJ, Bain G, Conder G, Hine AL, Misra RR: Tuberculosis: a radiologic review. RadioGraphics. 2007, 27:1255-1273. 10.1148/rg.275065176

10. Verver S, Warren RM, Beyers N, et al.: Rate of reinfection tuberculosis after successful treatment is higher than rate of new tuberculosis. Am J Respir Crit Care Med. 2005, 171:1430-1435. 10.1164/rccm.200409$12000 \mathrm{C}$

11. Yeh JJ, Yu JK, Teng WB, Chou CH, Hsieh SP, Lee TL, Wu MT: High-resolution CT for identify patients with smear-positive, active pulmonary tuberculosis. Eur J Radiol. 2012, 81:195-201. 10.1016/j.ejrad.2010.09.040

12. Bhalla AS, Goyal A, Guleria R, Gupta AK: Chest tuberculosis: radiological review and imaging recommendations. Indian J Radiol Imaging. 2015, 25:213-225. 10.4103/0971-3026.161431

13. Van Dyck P, Vanhoenacker FM, Van den Brande P, De Schepper AM: Imaging of pulmonary tuberculosis. Eur Radiol. 2003, 13:1771-1785. 10.1007/s00330-002-1612-y

14. Raghuvanshi V, Sood RG, Jhobta A, Sarkar M, Tomar A, Khanna S: Use of high-resolution computed tomography (HRCT) in diagnosis of sputum negative pulmonary tuberculosis. Turk Thorac J. 2016, 17:59-64. 10.5578/ttj.17.2.012

15. Alsowey AM, Amin MI, Said AM: The predictive value of multidetector high resolution computed tomography in evaluation of suspected sputum smear negative active pulmonary tuberculosis in Egyptian Zagazig University Hospital patients. Pol J Radiol. 2017, 82:808-816. 10.12659/PJR.903743

16. Lee HM, Shin JW, Kim JY, et al.: HRCT and whole-blood interferon-gamma assay for the rapid diagnosis of smear-negative pulmonary tuberculosis. Respiration. 2010, 79:454-460. 10.1159/000277926

17. Rizzi EB, Schinina' V, Cristofaro M, et al.: Detection of pulmonary tuberculosis: comparing MR imaging with HRCT. BMC Infect Dis. 2011, 11:243. 10.1186/1471-2334-11-243

18. Wekesa C, Kirenga BJ, Joloba ML, Bwanga F, Katamba A, Kamya MR: Chest X-ray vs Xpert ${ }^{\circledR}$ MTB/RIF assay for the diagnosis of sputum smear-negative tuberculosis in Uganda. Int J Tuberc Lung Dis. 2014, 18:216219. 10.5588/ijtld.13.0464 